\section{Crisis resolution and home treatment teams and intensive home treatment teams are worthwhile - but not everywhere}

On behalf of my co-authors, I thank the correspondents for their interest in our paper.

The Edinburgh Crisis Centre is undoubtedly an important resource for the city, but it is not a crisis house in the Camden mould. They have a maximum of four beds, with a maximum duration of stay of 7 days. They do not take referrals from the National Health Service (NHS), and do not share information with the NHS. During a 12-month period, they had only 12 residential clients, who were also working with our intensive home treatment team (IHTT). So to conclude that the Crisis Centre is the reason for a reduction in hospital admissions is simply not accurate, although IHTT values the presence of the Centre.

Dr Bhattacharya will have noted that we demonstrated a $17 \%$ reduction in admissions requiring detention during our study (see previous letter by Bhattacharya \& McQueen). This is in contrast to Forbes et al and Tyrer et al (see Bhattacharya \& McQueen for references). The Forbes study was based on a nurse-led service operating in a semi-rural environment, which already had a good-quality community mental health team and low base rate of detention. We have already mentioned the limitations of the Tyrer study. ${ }^{1}$ An important point about not conflating crisis teams with home treatment teams is also made, and we believe it is home treatment that can obviate the need for admission.

Finally, Drs Casserly \& Palin ${ }^{2}$ quite rightly suggest that our findings or model cannot automatically be generalised to other areas - this may be particularly true in remote or rural areas like Grampian. However, the planned bed closures they allude to would not have occurred without adequate alternative community provision - this was explicit in the strategy. Of course, once beds are closed, raw admission numbers fall, but not necessarily re-admissions or detentions (as we found). Further, mean length of stay has also fallen, consistent with a supportive 'early discharge' role. Naturally, we see a lot of dual diagnosis, but record only primary diagnosis. Last, pollsters such as MORI state that any postal survey with a response rate $>10 \%$ is valid, and $29 \%$ of over 700 cases is a reasonable return, with many patients stating that they preferred home to the local psychiatric hospital as their locus of care. It should also be noted that in over 2 years of IHTT working with individuals who are by definition high risk, only one suicide has (tragically) occurred.

So, even in these austere times service innovation can have positive outcomes, but it is important to critically appraise these innovations against existing practice.

1 Barker V, Taylor M, Kader I, Stewart K, Le Fevre P. Impact of crisis resolution and home treatment services on user experience and admission to psychiatric hospital. Psychiatrist 2011; 35 106-10

2 Casserly SM, Palin A. CRHT services and in-patient bed closured: the whole story? (e-letter). Psychiatrist 2011; 19 April (http:// pb.rcpsych.org/cgi/eletters/35/3/106\#10826).

Mark Taylor is a consultant psychiatrist, Intensive Home Treatment Team, Ballenden House, Edinburgh EH8 9HL, UK, email: marktaylor2@nhs.net

doi: $10.1192 / \mathrm{pb} .35 .7 .276$

\section{For whose benefit?}

Papers highlighting the difficult issue of 'off-label prescribing' are always of interest.' However, in the context of financial restraints and increasing cutbacks, the assertion, taken from the Royal College of Psychiatrists' guidelines, that such a modus operandi of prescribing requires frequent monitoring may be unhelpful. ${ }^{2}$

Clearly, several combinations of treatments for depression and other conditions might be 'off-label', yet they continue to be used regularly. Further, they are documented in widely known prescribing protocols such as the Maudsley guidelines and CANMET-MD, some with an arguably more transparent basis than the National Institute for Health and Clinical Excellence process for formulating guidelines. It might be time for psychiatrists to start using clinically appropriate, positiverisk 'off-label' prescribing that, as long as an evidence base has been followed, allows for the time interval between reviews to be increased. We should prescribe what makes a difference for the patient; not what contains the prescriber's anxiety.

1 Uzoechina CE, Hilvering P, Rogers C, O'Brien S, Pullela A, Yousafzai M, et al. Off-label prescribing in people with recurrent depressive disorder attending a community mental health service. Psychiatrist 2011; 35 84-9.

2 Holloway F. 'Gentlemen, we have no money therefore we must think' mental health services in hard times. Psychiatrist 2011; 35: 81-3.

Michael Jan Wise is consultant psychiatrist, Central and North West London NHS Foundation Trust, London NW6 6BX, UK, email: jan.wise@nhs.net

doi: 10.1192/pb.35.7.276a

\section{Emotional intelligence in psychiatrists and surgeons: issue of gender bias?}

Stanton et al present an interesting paper looking at the comparative analysis of emotional intelligence between psychiatrists and surgeons, but what I really want to know is whether there was any evidence of a difference in constituents (subscales) of emotional intelligence between genders? The reason I am raising this question is because there are gender differences found for the main factors that comprise emotional intelligence. $^{2}$ More specifically, women are more aware of their emotions, show more empathy, relate better interpersonally, and act in a more socially responsible manner than men. On the other hand, men appear to have better self-regard, are more independent, solve problems better, are more flexible, and cope better with stress. ${ }^{3}$

This is of great relevance seeing that there was a clear evidence of imbalance of gender distribution in Stanton et al's study: there were more female psychiatrists (39\%) as compared with female surgeons (17\%). This raises the possibility of a bias in relation to differences detected among psychiatrists and surgeons in the component factors that make up the total emotional intelligence. It is possible that a proportion of these differences detected among the two groups could be accounted for by gender imbalance. Future studies are needed to address this issue.

1 Stanton C, Sethi FN, Dale O, Phelan M, Laban JT, Eliahoo J. Comparison of emotional intelligence between psychiatrists and surgeons. Psychiatrist 2011; 35: 124-9. 